\title{
A boundary element approach to buckling of general laminates
}

\author{
S. Syngellakis \\ Wessex Institute of Technology, UK
}

\begin{abstract}
Laminates, comprising plies stacked in various orientations relative to their principal material frames of reference, generally exhibit coupling between their in-plane displacements and transverse deflection under any loading conditions. The main theme of this paper is the development of integral equations for the bifurcation buckling analysis of laminates taking into account this coupling. The formulation of the extensional problem is based on the stress function concept, which results in constitutive relations and a field equation mathematically equivalent to those of the plate bending problem. This has the advantage of using the same form of fundamental solution, similar boundary and domain modelling as well as the development of common algorithms for the solution of the two coupled problems. Modelling approaches for dealing with irreducible domain integrals arising from both extension-flexure coupling and geometric nonlinearity are presented.

Keywords: laminates, bending-extension coupling, buckling, boundary elements.
\end{abstract}

\section{Introduction}

The design of structural components made of composites can be tailored to meet the specific demands of a particular application by making optimum use of available materials. The mechanical behaviour of flat laminates, in particular, depends on ply orientation, number of plies in a given direction, thickness of individual plies, type of ply and the ply stacking sequence. The latter generally causes coupling between the transverse deflection and the in-plane displacements of a laminate. This coupling is absent only in the special case of "balanced" laminates in which plies are symmetrically arranged with respect to the middle plane of the plate. The coupling effects have been taken into account in the 
determination of the exact buckling load of a particular, angle-ply type of laminates [1]. Due however to the complexity of the general laminate behaviour, the use of advanced numerical techniques was found necessary for their analysis; their buckling and post-buckling behaviour has been modelled using the finite element [2], the Rayleigh-Ritz [3], and the Galerkin method [4].

The boundary element method (BEM) is another numerical tool through which the dimensionality of a problem can be reduced by one and mesh generation becomes comparatively easy. Existing BEM formulations, based on the generalised Rayleigh-Green identity for anisotropic plates, predict the linear flexural response of balanced laminates with any plan form and a number of corner points along their boundary [5]. The same direct BEM approach was applied to both in-plane and flexural analysis of balanced laminates using the Airy's stress function and the deflection as field variables [6]. Initial BEM work on buckling was restricted to orthotropic plates [7, 8]. Linear and nonlinear buckling analyses of anisotropic balanced laminates under any in-plane force distribution were subsequently developed and implemented $[9,10]$. Fundamental solutions have recently been obtained and boundary integral equations formulated for the coupled extension-flexure of general laminates $[11,12]$.

The BEM approach adopted in the present paper models the critical buckling behaviour of general anisotropic laminates as represented by the classical lamination theory. The formulation uses the fundamental solution for the linear, uncoupled plate extensional or flexural problem. The resulting integral equations contain irreducible domain integrals depending on terms arising from the geometric nonlinearity as well as extension-flexure coupling. The paper concludes with a discussion on possible techniques for modelling and evaluation of these irreducible domain integrals.

\section{Nonlinear laminate theory}

According to the classical lamination theory, the plate is assumed to consist of an arbitrary number of discrete layers, each individual layer being homogeneous through its thickness and in a state of plane stress. The laminate is also assumed to deform according to Kirchhoff's bending theory of thin plates; as a consequence, strains $\varepsilon_{\alpha \beta}$ and curvatures $\kappa_{\alpha \beta}$ are related to mid-plane displacements $u_{\alpha}$ and deflection $w$ by

$$
\begin{gathered}
\varepsilon_{\alpha \beta}=\frac{1}{2}\left(u_{\alpha, \beta}+u_{\beta, \alpha}+w,_{\alpha} w_{, \beta}\right) \\
\kappa_{\alpha \beta}=-w, \alpha \beta
\end{gathered}
$$

where the Greek indices range from 1 to 2 indicating components relative to a Cartesian, $x_{1}-x_{2}$ frame of reference and a comma followed by a lower index indicates differentiation with respect to the corresponding co-ordinate. Membrane forces $N_{\alpha \beta}$ and bending moments $M_{\alpha \beta}$ are related to the strains and curvatures by

$$
\begin{aligned}
& N_{\alpha \beta}=A_{\alpha \beta \gamma \delta} \varepsilon_{\gamma \delta}+B_{\alpha \beta \gamma \delta} \kappa_{\gamma \delta} \\
& M_{\alpha \beta}=B_{\alpha \beta \gamma \delta} \varepsilon_{\gamma \delta}+D_{\alpha \beta \gamma \delta} \kappa_{\gamma \delta}
\end{aligned}
$$


where $A_{\alpha \beta \gamma \delta}$ and $D_{\alpha \beta \gamma \delta}$ are, respectively, the extensional and flexural rigidities, $B_{\alpha \beta \gamma \delta}$ are extensional-flexural coupling coefficients and repeated indices mean summation over their range. The governing field equations and the boundary conditions can be obtained by minimising the potential energy functional

$$
\Pi=1 / 2 \int_{\Omega}\left(N_{\alpha \beta} \varepsilon_{\alpha \beta}+M_{\alpha \beta} \kappa_{\alpha \beta}\right) \mathrm{d} \Omega-\int_{\Gamma} \tilde{p}_{\alpha} u_{\alpha} \mathrm{d} \Gamma
$$

where $\tilde{p}_{\alpha}$ is the prescribed edge traction, $\Omega$ the plate domain bounded by the contour $\Gamma$, as shown in Fig. 1. The normal and tangent unit vectors to $\Gamma$ are represented by $\mathbf{n}$ and $\mathbf{s}$, respectively, and the path variable along $\Gamma$ by $s ; \Gamma$ is smooth apart from a finite number of corner points at $s_{j}, j=1, \ldots, K$.

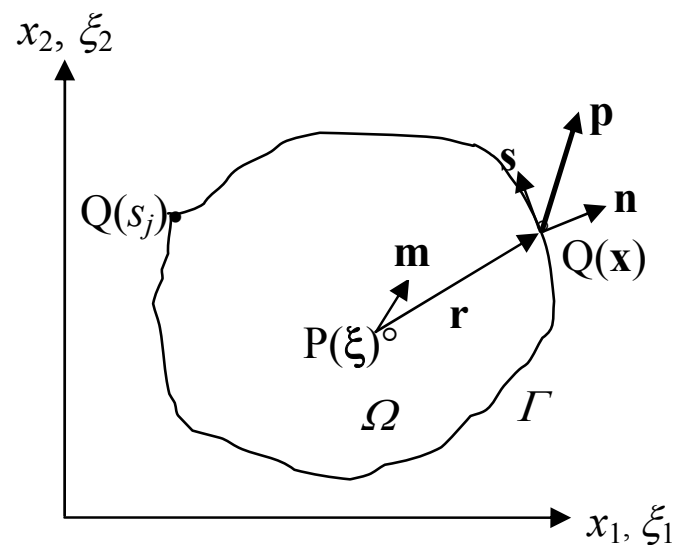

Figure 1: Plate schematic with relevant notation.

\section{Linear pre-buckling state}

Buckling is due to the action of in-plane tractions $\lambda \tilde{p}_{\alpha}$ where $\tilde{p}_{\alpha}$ is some reference load causing the linear pre-buckling state $\left[N_{\alpha \beta}^{0}, M_{\alpha \beta}^{0}\right]$. The unloaded plate is assumed to be perfectly flat and it buckles at a critical value of the load factor $\lambda$. As with the general non-linear problem, the governing equations for the pre-buckling state are obtained from the variational principle $\delta \Pi^{0}=0$ where $\delta$ is the variation symbol. The membrane forces $N_{\alpha \beta}^{0}$ and bending moments $M_{\alpha \beta}^{0}$ are thus found to satisfy the equations of equilibrium

$$
\begin{gathered}
N_{\alpha \beta}^{0},_{\beta}=0 \\
M_{\alpha \beta}^{0},_{\alpha \beta}=0
\end{gathered}
$$

Along the smooth portions of $\Gamma$, the field variables should satisfy the boundary conditions

$$
\begin{gathered}
p_{\alpha}^{0}=\tilde{p}_{\alpha} \\
V_{n}^{0}=0 \text { or } w^{0}=0
\end{gathered}
$$




$$
M_{n}^{0}=0 \text { or } \theta_{n}^{0}=0
$$

while, at any corner point co-ordinate $s_{j}$,

$$
C_{j}^{0}=0 \text { or } w_{j}^{0}=0
$$

where the boundary variables are related to the domain variables by

$$
\begin{gathered}
p_{\alpha}^{0}=n_{\beta} N_{\alpha \beta}^{0} \\
V_{n}^{0}=n_{\alpha} M_{\alpha \beta}^{0},_{\beta}+\frac{\partial M_{n s}^{0}}{\partial s} \\
M_{n}^{0}=n_{\alpha} n_{\beta} M_{\alpha \beta}^{0}
\end{gathered}
$$

and $C_{j}^{0}=M_{n s}^{0}\left(s_{j}^{+}\right)-M_{n s}^{0}\left(s_{j}^{-}\right)$represents the discontinuity jump of the twisting moment:

$$
M_{n s}^{0}=s_{\alpha} n_{\beta} M_{\alpha \beta}^{0}
$$

The problem for the membrane forces can be re-formulated in terms of a stress function $F$ such that

$$
N_{\alpha \beta}^{0}=L_{\alpha \beta} F^{0}
$$

with the operator $L_{\alpha \beta}$ defined by

$$
L_{\alpha \beta}=\delta_{\alpha \beta} \nabla^{2}-\frac{\partial^{2}}{\partial x_{\alpha} \partial x_{\beta}}
$$

and $\delta_{\alpha \beta}$ representing the Kronecker delta. In-plane equilibrium eqns (6) are identically satisfied if the membrane forces are given by expressions (12). Referring to fig. 1, it is possible to show that, at any point $Q\left(\bar{x}_{1}, \bar{x}_{2}\right)$ along the boundary of the plate,

$$
\begin{gathered}
F^{0}=\int_{O}^{Q}\left[\left(x_{1}-\bar{x}_{1}\right) \tilde{p}_{2}-\left(x_{2}-\bar{x}_{2}\right) \tilde{p}_{1}\right] \mathrm{d} \Gamma \\
\frac{\partial F^{0}}{\partial n}=-s_{1}(Q) \int_{O}^{Q} \tilde{p}_{1} \mathrm{~d} \Gamma-s_{2}(Q) \int_{O}^{Q} \tilde{p}_{2} \mathrm{~d} \Gamma
\end{gathered}
$$

where $O$ is an arbitrarily located origin for the path variable along $\Gamma$. According to eqn (14), $F^{0}$ can be physically interpreted as the resultant moment about $Q$ of the traction over $O Q$. Similarly, eqn (15) describes the normal derivative of $F^{0}$ as the component of the resultant traction over $O Q$ in the direction $-\mathbf{s}$ at $Q$.

Omitting the nonlinear terms from eqn (1), it is easily shown that the linear pre-buckling strains satisfy the compatibility equation

$$
L_{\alpha \beta} \varepsilon_{\alpha \beta}^{0}=\bar{\varepsilon}_{\alpha \beta}^{0},_{\alpha \beta}=0
$$

where the strain tensor has been re-defined as

$$
\bar{\varepsilon}_{\alpha \beta}^{0}=\delta_{\alpha \beta} \varepsilon_{\kappa \kappa}^{0}-\varepsilon_{\alpha \beta}^{0}
$$

Constitutive equations (3) and (4) are next re-arranged so that $\bar{\varepsilon}_{\alpha \beta}^{0}$ is expressed in terms of $F^{0}$ and $w^{0}$. Defining $A_{\alpha \beta \gamma \delta}^{-1}$ as the inverse of the extensional rigidity tensor $A_{\alpha \beta \gamma \delta}$,

$$
A_{\alpha \beta \gamma \delta}^{-1} A_{\gamma \delta \lambda \mu}=\delta_{\alpha \lambda} \delta_{\beta \mu}
$$


it is possible to show through a series of substitutions that

$$
\begin{aligned}
\bar{\varepsilon}_{\alpha \beta}^{0} & =\hat{A}_{\alpha \beta \gamma \delta} F^{0}{ }_{\gamma \delta}+\hat{B}_{\alpha \beta \gamma \delta} w^{0}{ }_{\gamma \delta} \\
M_{\alpha \beta}^{0} & =\hat{B}_{\gamma \delta \alpha \beta} F^{0}{ }_{, \gamma \delta}-\hat{D}_{\alpha \beta \gamma \delta} w^{0}{ }_{\gamma \delta}
\end{aligned}
$$

where

$$
\begin{gathered}
\hat{A}_{\alpha \beta \gamma \delta}=A_{\kappa \kappa \lambda \lambda}^{-1} \delta_{\alpha \beta} \delta_{\gamma \delta}-A_{\alpha \beta \kappa \kappa}^{-1} \delta_{\gamma \delta}-A_{\kappa \kappa \gamma \delta}^{-1} \delta_{\alpha \beta}+A_{\alpha \beta \gamma \delta}^{-1} \\
\hat{B}_{\alpha \beta \gamma \delta}=\left(A_{\kappa \kappa \lambda \mu}^{-1} \delta_{\alpha \beta}-A_{\alpha \beta \lambda \mu}^{-1}\right) B_{\lambda \mu \gamma \delta}
\end{gathered}
$$

and

$$
\hat{D}_{\alpha \beta \gamma \delta}=D_{\alpha \beta \gamma \delta}-B_{\alpha \beta \kappa \lambda} A_{\kappa \lambda \mu \nu}^{-1} B_{\mu v \gamma \delta}
$$

is the reduced flexural stiffness tensor. Substitution of the new constitutive eqns (18) and (19) into the compatibility eqn (16) and equilibrium eqn (7) leads to the differential field equations

$$
\begin{gathered}
\hat{A}_{\alpha \beta \gamma \delta} F^{0}{ }_{\alpha \beta \gamma \delta}+\hat{B}_{\alpha \beta \gamma \delta} w^{0}{ }_{\alpha \beta \gamma \delta}=0 \\
\hat{B}_{\gamma \delta \alpha \beta} F^{0}{ }_{\alpha \beta \gamma \delta}-\hat{D}_{\alpha \beta \gamma \delta} w^{0}{ }_{\alpha \beta \gamma \delta}=0
\end{gathered}
$$

\section{Bifurcation buckling}

The pre-buckling state of equilibrium becomes unstable at a critical value $\lambda_{c} \tilde{p}_{\alpha}$ of the factored edge load. Then a second equilibrium state exists, associated with inplane displacements $u_{\alpha}^{1}\left(x_{\alpha}\right)$ and a lateral deflection $w^{1}\left(x_{\alpha}\right)$. According to the general theory of elastic stability, the differential equation and boundary conditions governing the buckling mode $\left(u_{\alpha}^{1}, w^{1}\right)$ can be obtained from a variational principle [13], which, in the present unbalanced laminate case, takes the form

$$
\int_{\Omega}\left(N_{\alpha \beta}^{1} \delta u_{\alpha},{ }_{\beta}-M_{\alpha \beta}^{1} \delta w_{\alpha \beta}\right) \mathrm{d} \Omega+\lambda_{c} \int_{\Omega}\left(N_{\alpha \beta}^{1} w^{0},{ }_{\alpha}+N_{\alpha \beta}^{0} w^{1},{ }_{\alpha}\right) \delta w_{\beta} \mathrm{d} \Omega=0
$$

The constitutive relations for the buckling mode are the same as those for the pre-buckling state with the strains $\varepsilon_{\alpha \beta}^{1}$ and curvatures $\kappa_{\alpha \beta}^{1}$ given by

$$
\begin{gathered}
\varepsilon_{\alpha \beta}^{1}=\frac{1}{2}\left[u_{\alpha}^{1},,_{\beta}+u_{\beta}^{1},_{\alpha}+\lambda_{c}\left(w^{0},_{\alpha} w^{1},{ }_{\beta}+w^{0},_{\beta} w^{1},{ }_{\alpha}\right)\right] \\
\kappa_{\alpha \beta}^{1}=-w^{1},_{\alpha \beta}
\end{gathered}
$$

Elimination of displacement from eqn (25), leads to the compatibility condition

$$
L_{\alpha \beta} \varepsilon_{\alpha \beta}^{1}+\lambda_{c}\left(L_{\alpha \beta} w^{0}\right) w^{1},_{\alpha \beta}=\bar{\varepsilon}_{\alpha \beta}^{1},_{\alpha \beta}+\lambda_{c}\left(L_{\alpha \beta} w^{0}\right) w^{1},_{\alpha \beta}=0
$$

The condition that Eq. (24) be satisfied for arbitrary $\left(\delta u_{\alpha}, \delta w\right)$ yields the field equations

$$
\begin{gathered}
N_{\alpha \beta}^{1},_{\beta}=0 \\
M_{\alpha \beta}^{1},_{\alpha \beta}+\lambda_{c}\left(N_{\alpha \beta}^{1} w^{0},{ }_{\alpha \beta}+N_{\alpha \beta}^{0} w^{1},{ }_{\alpha \beta}\right)=0
\end{gathered}
$$

over domain $\Omega$, the boundary conditions

$$
p_{\alpha}^{1}=0
$$




$$
\begin{gathered}
\text { either } M_{n}^{1}=0 \text { or } \theta_{n}^{1}=0 \\
\text { either } V_{n}^{1}+\lambda_{c} p_{\alpha}^{0} w^{1},{ }_{\alpha}=0 \text { or } w^{1}=0
\end{gathered}
$$

on $\Gamma$ and

$$
\text { either } C_{j}^{1}=0 \text { or } w_{j}^{1}=0
$$

at the corner co-ordinates $s_{j}, j=1, \ldots, K$.

The membrane forces $N_{\alpha \beta}^{1}$ identically satisfy equilibrium eqns (28) if they are also expressed in terms of a stress function $F^{1}$ according to eqn (12). It is evident from eqns (14) and (15) and boundary condition (30) that

$$
F^{1}=\frac{\partial F^{1}}{\partial n}=0
$$

on $\Gamma$. Since constitutive equations (3) and (4) relate the membrane forces $N_{\alpha \beta}^{1}$ and bending moments $M_{\alpha \beta}^{1}$ to the respective strains and curvatures, they can be re-arranged in the same manner as those for the pre-buckling state to give

$$
\begin{gathered}
\bar{\varepsilon}_{\alpha \beta}^{1}=\hat{A}_{\alpha \beta \gamma \delta} F^{1}{ }_{\gamma \delta}+\hat{B}_{\alpha \beta \gamma \delta} w^{1}{ }_{\gamma \delta} \\
M_{\alpha \beta}^{1}=\hat{B}_{\gamma \delta \alpha \beta} F^{1}{ }_{\gamma \delta}-\hat{D}_{\alpha \beta \gamma \delta} w^{1}{ }_{\gamma \delta}
\end{gathered}
$$

Thus eqn (27) together with eqn (29) are transformed to

$$
\begin{gathered}
\hat{A}_{\alpha \beta \gamma \delta} F^{1},_{\alpha \beta \gamma \delta}+\hat{B}_{\alpha \beta \gamma \delta} w^{1},_{\alpha \beta \gamma \delta}+\lambda_{c}\left(L_{\alpha \beta} w^{0}\right) w^{1},_{\alpha \beta}=0 \\
\hat{B}_{\gamma \delta \alpha \beta} F^{1},_{\alpha \beta \gamma \delta}-\hat{D}_{\alpha \beta \gamma \delta} w^{1}{ }_{\alpha \beta \gamma \delta}+\lambda_{c}\left(N_{\alpha \beta}^{1} w^{0},_{\alpha \beta}+N_{\alpha \beta}^{0} w^{1},_{\alpha \beta}\right)=0
\end{gathered}
$$

that is, the coupled linear field equations governing the buckling mode of unbalanced laminates subjected to in-plane forces.

\section{Integral equations}

The two coupled problems for the pre-buckling state and those for the buckling mode can be solved by a common BEM approach based on the Rayleigh-Green identity and the fundamental solutions for the fourth order linear symmetric operator

$$
C_{\alpha \beta \gamma \delta} \frac{\partial^{4}}{\partial x_{\alpha} \partial x_{\beta} \partial x_{\gamma} \partial x_{\delta}}
$$

where $C_{\alpha \beta \gamma \delta}$ can either be the modified extensional compliance tensor $\hat{A}_{\alpha \beta \gamma \delta}$ or the reduced flexural rigidity tensor $\hat{D}_{\alpha \beta \gamma \delta}$. The generic fundamental solutions $U_{\lambda}^{*}(\lambda=1,2)$ for operator $(38)$ satisfy

$$
C_{\alpha \beta \gamma \delta} U_{\lambda}^{*},_{\alpha \beta \gamma \delta}=\delta_{\lambda}(\mathbf{x}-\xi) ; \lambda=1,2
$$

where $\delta_{\lambda}$ is related to the Dirac delta function $\delta$ according to

$$
\begin{gathered}
\delta_{1}(\mathbf{x}-\xi)=\delta(\mathbf{x}-\xi) \\
\delta_{2}(\mathbf{x}-\xi)=\frac{\partial \delta(\mathbf{x}-\xi)}{\partial m(\xi)}
\end{gathered}
$$


and $m$ represents an arbitrary direction through the source point as shown in fig. 1. The symmetry of $C_{\alpha \beta \gamma \delta}$ with respect to the pairs of indices $(\alpha \beta)$ and $(\gamma \delta)$ leads to the reciprocity relation

$$
\int_{\Omega} C_{\alpha \beta \gamma \delta} U,{ }_{\gamma \delta} U_{\lambda,{ }_{\alpha \beta}}^{*} \mathrm{~d} \Omega=\int_{\Omega} C_{\alpha \beta \gamma \delta} U,_{\alpha \beta} U_{\lambda,{ }_{\gamma \delta}}^{*} \mathrm{~d} \Omega
$$

where $U$ is any real smooth function over the domain $\Omega$. A compact form of $U_{\lambda}^{*}$ as well as the various kernels derived from it can be found in the literature [6].

\subsection{Pre-buckling}

With $C_{\alpha \beta \gamma \delta}=\hat{A}_{\alpha \beta \gamma \delta}$ and the pair of functions $F^{0}$ and $F_{\lambda}^{*}$ replacing $U$ and $U_{\lambda}^{*}$, eqn (42) is transformed to

$$
\int_{\Omega}\left(\bar{\varepsilon}_{\alpha \beta}^{0}-\hat{B}_{\alpha \beta \gamma \delta} w^{0},_{\gamma \delta}\right) F_{\lambda}^{*},_{\alpha \beta} \mathrm{d} \Omega=\int_{\Omega} \hat{A}_{\alpha \beta \gamma \delta} F_{\lambda}^{*},_{\gamma \delta} F^{0},_{\alpha \beta} \mathrm{d} \Omega
$$

Integrations by parts and repeated application of Green's theorem transform eqn (43) into

$$
k F_{\lambda}^{0}\left(\xi_{\alpha}\right)=I_{b}^{A}\left(F^{0}, F_{\lambda}^{*}\right)+J^{A}\left(F^{0}, F_{\lambda}^{*}\right)-\int_{\Omega}\left(\hat{B}_{\alpha \beta \gamma \delta} w^{0},_{\gamma \delta}\right) F_{\lambda}^{*},_{\alpha \beta} \mathrm{d} \Omega
$$

where $F_{1}^{0}=F^{0}$,

$$
k=1, F_{2}^{0}=\frac{\partial F^{0}}{\partial m}
$$

if $P\left(\xi_{\alpha}\right)$ is a point in the domain, or

$$
k=0.5, F_{2}^{0}=\frac{\partial F^{0}}{\partial n}
$$

if $P\left(\xi_{\alpha}\right)$ lies on a smooth portion of the boundary and

$$
\begin{aligned}
I_{b}^{A}\left(F^{0}, F_{\lambda}^{*}\right)= & \int_{\Gamma}\left(\frac{\partial^{2} u_{n}^{0}}{\partial s^{2}} F_{\lambda}^{*}+\varepsilon_{s s}^{0} \frac{\partial F_{\lambda}^{*}}{\partial n}-\varepsilon_{\lambda s s}^{*} \frac{\partial F^{0}}{\partial n}-\frac{\partial^{2} u_{\lambda n}^{*}}{\partial s^{2}} F^{0}\right) \mathrm{d} \Gamma \\
& J^{A}\left(F^{0}, F_{\lambda}^{*}\right)=\sum_{j=1}^{K} \llbracket \varepsilon_{n s}^{0} F_{\lambda}^{*}-\varepsilon_{\lambda n s}^{*} F^{0} \rrbracket_{j}
\end{aligned}
$$

The boundary variables $\varepsilon_{s s}^{0}, \varepsilon_{n s}^{0}$ and $u_{n}^{0}$ represent real boundary strains and displacement, while the kernels appearing in eqns (45) and (46) are artificial entities obtainable from

$$
\begin{gathered}
\varepsilon_{\lambda s s}^{*}=\hat{A}_{\alpha \beta \gamma \delta} n_{\alpha} n_{\beta} F_{\lambda,{ }_{\gamma \delta}^{*}}^{*} \\
\varepsilon_{\lambda n s}^{*}=-\hat{A}_{\alpha \beta \gamma \delta} s_{\alpha} n_{\beta} F_{\lambda, \gamma \delta}^{*} \\
\frac{\partial^{2} u_{\lambda n}^{*}}{\partial s^{2}}=-\hat{A}_{\alpha \beta \gamma \delta} n_{\alpha} F_{\lambda}^{*},_{\beta \gamma \delta}+\frac{\partial \varepsilon_{\lambda n s}^{*}}{\partial s}
\end{gathered}
$$

In the case of flexure, $\hat{D}_{\alpha \beta \gamma \delta}$ replaces $C_{\alpha \beta \gamma \delta}$ and the pair of functions $w$ and $w_{\lambda}^{*}$ replace $U$ and $U_{\lambda}^{*}$ in the reciprocity relation (42), which thus becomes

$$
\int_{\Omega}\left(M_{\alpha \beta}^{0}-\hat{B}_{\gamma \delta \alpha \beta} F^{0},_{\gamma \delta}\right) w_{\lambda}^{*},_{\alpha \beta} \mathrm{d} \Omega=\int_{\Omega} M_{\lambda \alpha \beta}^{*} w^{0},_{\alpha \beta} \mathrm{d} \Omega
$$


A similar process to that applied to eqn (43) also transforms eqn (50) to the integral equation

$$
k w_{\lambda}^{0}\left(\xi_{\alpha}\right)=I_{b}^{D}\left(w^{0}, w_{\lambda}^{*}\right)+J^{D}\left(w^{0}, w_{\lambda}^{*}\right)+\int_{\Omega} \hat{B}_{\gamma \delta \alpha \beta} w_{\lambda}^{*},_{\alpha \beta} F^{0},_{\gamma \delta} \mathrm{d} \Omega
$$

where $w_{1}=w^{0}$,

$$
w_{2}=\frac{\partial w^{0}}{\partial m} \text { or } w_{2}=\frac{\partial w^{0}}{\partial n}
$$

depending on whether $P$ is in the domain or on smooth portion of the boundary, respectively, and

$$
\begin{gathered}
I_{b}^{D}\left(w^{0}, w_{\lambda}^{*}\right)=\int_{\Gamma}\left(V_{n}^{0} w_{\lambda}^{*}-M_{n}^{0} \frac{\partial w_{\lambda}^{*}}{\partial n}+M_{\lambda n}^{*} \frac{\partial w^{0}}{\partial n}-V_{\lambda n}^{*} w^{0}\right) \mathrm{d} \Gamma \\
J^{D}\left(w^{0}, w_{\lambda}^{*}\right)=\sum_{j=1}^{K} \llbracket M_{n s}^{0} w_{\lambda}^{*}-M_{\lambda n s}^{*} w^{0} \rrbracket_{j}
\end{gathered}
$$

The kernels in the boundary integral and the jump term are obtained from

$$
\begin{gathered}
M_{\lambda n}^{*}=-\hat{D}_{\alpha \beta \gamma \delta} n_{\alpha} n_{\beta} w_{\lambda}^{*},_{\gamma \delta} \\
M_{\lambda n s}^{*}=-\hat{D}_{\alpha \beta \gamma \delta} s_{\alpha} n_{\beta} w_{\lambda, \gamma \delta}^{*} \\
V_{\lambda n}^{*}=-\hat{D}_{\alpha \beta \gamma \delta} n_{\alpha} w_{\lambda}^{*},_{\beta \gamma \delta}+\frac{\partial M_{\lambda n s}^{*}}{\partial s}
\end{gathered}
$$

The irreducible domain integrals

$$
\int_{\Omega}\left(\hat{B}_{\alpha \beta \gamma \delta} w^{0},_{\gamma \delta}\right) F_{\lambda}^{*},_{\alpha \beta} \mathrm{d} \Omega \text { and } \int_{\Omega}\left(\hat{B}_{\gamma \delta \alpha \beta} F^{0},_{\gamma \delta}\right) w_{\lambda}^{*},_{\alpha \beta} \mathrm{d} \Omega,
$$

arising from extensional-flexural mode coupling, depend on the second partial derivatives of the stress function and the deflection, representing membrane forces and curvatures, respectively. The numerical solution of integral equations (44) and (51) largely depends on the possibility of extending the usual BE methodology to include domain variables and the evaluation of the irreducible domain integrals. The scheme for dealing with these integrals can be similar to that adopted for three-dimensional anisotropic elasticity [14]. With the source point in the domain $(k=1)$, eqns (44) and (51) are differentiated twice with respect to the source variable $\xi_{\alpha}$ to give two supplementary integral equations

$$
\begin{gathered}
F^{0},_{\mu \nu}=I_{b}^{A}\left(F^{0}, F_{\lambda}^{*},_{\mu \nu}\right)+J^{A}\left(F^{0}, F_{\lambda}^{*},_{\mu \nu}\right)-\int_{\Omega}\left(\hat{B}_{\alpha \beta \gamma \delta} w^{0},_{\gamma \delta}\right) F_{\lambda}^{*},_{\alpha \beta \mu \nu} \mathrm{d} \Omega \\
w^{0},_{\mu \nu}=I_{b}^{D}\left(w^{0}, w_{\lambda}^{*},_{\mu \nu}\right)+J^{D}\left(w^{0}, w_{\lambda}{ }_{\lambda},_{\mu \nu}\right)+\int_{\Omega}\left(\hat{B}_{\gamma \delta \alpha \beta} F^{0},_{\gamma \delta}\right) w_{\lambda}^{*},_{\alpha \beta \mu \nu} \mathrm{d} \Omega
\end{gathered}
$$

It should be noted that, in contrast to the three-dimensional elasticity problem [14], there is no need for additional convection terms since $U_{\lambda}^{*},{ }_{\alpha \beta \mu \nu}$ is of order $\mathrm{O}\left(r^{-2}\right)$ and therefore integrable in $\Omega[15]$.

As with previous BE analyses of laminate buckling [9, 16], modelling can again be implemented using linear or quadratic discontinuous boundary elements and triangular domain cells. Thus, the derived integral equations are transformed into four systems of algebraic equations, which can be solved for the four arrays of discrete values of boundary and domain unknowns. It is more convenient to 
eliminate the boundary rather than the domain unknowns from these systems and solve for the domain forces and curvatures, which are eventually substituted into the equations governing the buckling mode and critical load factor.

\subsection{Buckling mode}

The boundary element formulation for the buckling mode is obtained by a similar procedure to that applied for the pre-buckling state. The initial reciprocity relations are similar to eqns (43) and (50) but upon integration by parts and the application of Green's theorem, they are transformed to the integral equations

$$
\begin{gathered}
k F_{\lambda}^{1}\left(\xi_{\alpha}\right)=I_{b}^{A}\left(F^{1}, F_{\lambda}^{*}\right)+J^{A}\left(F^{1}, F_{\lambda}^{*}\right) \\
-\int_{\Omega}\left(\hat{B}_{\alpha \beta \gamma \delta} w^{1},_{\gamma \delta}\right) F_{\lambda}^{*},_{\alpha \beta} \mathrm{d} \Omega-\lambda_{c} \int_{\Omega}\left(L_{\alpha \beta} w^{0}\right) w^{1},_{\alpha \beta} F_{\lambda}^{*} \mathrm{~d} \Omega \\
k w_{\lambda}^{1}\left(\xi_{\alpha}\right)=I_{b}^{D}\left(w^{1}, w_{\lambda}^{*}\right)+J^{D}\left(w^{1}, w_{\lambda}^{*}\right)+\int_{\Omega} \hat{B}_{\gamma \delta \alpha \beta} w_{\lambda}^{*}{ }_{\alpha \beta} F^{1},_{\gamma \delta} \mathrm{d} \Omega \\
+\lambda_{c} \int_{\Omega}\left(N_{\alpha \beta}^{1} w^{0},_{\alpha \beta}+N_{\alpha \beta}^{0} w^{1},_{\alpha \beta}\right) w_{\lambda}^{*} \mathrm{~d} \Omega
\end{gathered}
$$

Thus, in the case of buckling, domain integrals arise from both the extensionflexure coupling and geometric nonlinearity, which generates the additional terms in eqns (27) and (29); all these integrals depend on membrane forces and curvatures. Due to the presence of such domain integrals, eqns (59) and (60) are not proper boundary integral equations. As with the pre-buckling solution, it is possible again to generate two supplementary integral equations:

$$
\begin{gathered}
F^{1},_{\mu \nu}=I_{b}^{A}\left(F^{1}, F_{\lambda}^{*},_{\mu \nu}\right)+J^{A}\left(F^{1}, F_{\lambda}^{*},_{\mu \nu}\right) \\
-\int_{\Omega}\left(\hat{B}_{\alpha \beta \gamma \delta} w^{1},_{\gamma \delta}\right) F_{\lambda}^{*},_{\alpha \beta \mu \nu} \mathrm{d} \Omega-\lambda_{c} \int_{\Omega}\left(L_{\alpha \beta} w^{0}\right) w^{1},_{\alpha \beta} F_{\lambda}^{*},_{\mu \nu} \mathrm{d} \Omega \\
w^{1},_{\mu \nu}=I_{b}^{D}\left(w^{1}, w_{\lambda}^{*},_{\mu \nu}\right)+J^{D}\left(w^{1}, w_{\lambda}^{*},{ }_{\mu \nu}\right)+\int_{\Omega}\left(\hat{B}_{\gamma \delta \alpha \beta} F^{1}{ }_{, \gamma \delta}\right) w_{\lambda}^{*}{ }_{\alpha \beta \mu \nu} \mathrm{d} \Omega \\
+\lambda_{c} \int_{\Omega}\left(N_{\alpha \beta}^{1} w^{0},_{\alpha \beta}+N_{\alpha \beta}^{0} w^{1},_{\alpha \beta}\right) w_{\lambda}^{*},_{\mu \nu} \mathrm{d} \Omega
\end{gathered}
$$

from integral equations (59) and (60) through straightforward differentiation.

Modelling for the buckling mode can be performed in the same manner as for the pre-buckling analysis. There are three additional domain integrals to be calculated but the elements of all other coefficient matrices are the same as those for the pre-buckling solution. It is convenient again to eliminate the boundary unknowns from the derived system of algebraic equations and formulate a classical eigenvalue problem for the critical load factor $\lambda_{c}$ and the associated buckling mode $\left(F^{1}, w^{1}\right)$.

\section{Discussion}

The numerical integration schemes for the implementation of the proposed methodology can be similar to those adopted for the BEM buckling analysis of isotropic and orthotropic plates. Particular attention needs to be given to the evaluation of hyper-singular domain integrals generated by the repeated 
differentiation of the kernels of the domain integrals arising from extensionalflexural coupling. Special evaluation schemes of such integrals involving kernels with $\mathrm{O}\left(r^{-2}\right)$ singularity have been proposed and successfully applied in various BEM applications.

Numerical results are essential for the validation of the developed formulation but also for confirming that bifurcation buckling is possible in general laminates which are expected to undergo a gradual transition from an initial low-deflection to a large-deflection regime [1]. Since the pre-buckling state has been forced here to remain linear, bifurcation buckling is the only means of the laminate going into the large deflection regime. This is similar to a phenomenon known as secondary bifurcation observed in other stability problems such as that of initially imperfect stiffened shells [17]. The validated BEM solution can then be used to the systematic assessment of the coupling effects on the buckling of general laminates with various ply arrangements. The pre-buckling solution can also be used to predict more accurately the response of a general laminate to lateral loads. The BEM buckling formulation can be subsequently extended to the nonlinear, large deformation of unbalanced laminates, which will provide information on their post-buckling stiffness.

An alternative to the generation of additional integral equations and 6 domain variables per node is to adopt higher-order interpolation models for the stress function and the deflection, which are then entered into eqns (2) and (12) to generate the nodal in-plane forces and curvatures. Additional systems of equations can be obtained by applying integral eqns (44) and (51) with $k=\lambda=1$, that is, with the source point placed on the domain nodes. In-plane forces and curvatures can thus be eliminated from the final system of equations, which would contain only the stress function and the deflection as well as the associated boundary variables as unknowns. This approach has been tested in the case of isotropic plates [18] and can be modified by adopting a meshless modelling strategy, possibly combined with dual reciprocity schemes to generate a pure BEM formulation.

\section{References}

[1] Harris, G.Z., Buckling and post-buckling behaviour of composite plates under biaxial loading. International Journal of Mechanical Sciences, 17(3), pp. 187-202, 1975.

[2] Noor, A.K., Mathers, M.D. and Anderson, M.S., Exploiting symmetries for efficient postbuckling analysis of composite plates. AIAA Journal, 15(1), pp. 24-32, 1976.

[3] Minguet, P.J., Dugundji, J. and Lagace, P., Postbuckling behavior of laminated plates using a direct energy-minimization technique. AIAA Journal, 27(12), pp. 1785-1792, 1989.

[4] Prabhakara, M.K., Buckling of Laminated Composite Plates and Shell Panels with Some Free Edges under Compression and Shear. Aeronautical Journal, 96(951), pp. 20-26, 1992. 
[5] Shi, G. and Bezine, G., A general boundary integral formulation for the anisotropic plate bending problems. Journal of Composite Materials, 22, pp. 694-716, 1988.

[6] Syngellakis, S. and Cherukunnath, N., Boundary element analysis of symmetrically laminated plates. Engineering Analysis with Boundary Elements, 28(9), pp. 1005-1016, 2004.

[7] Shi, G. and Bezine, G., Buckling analysis of orthotropic plates by boundary element method. Mechanics Research Communications, 17(1), pp. 1-8, 1990.

[8] Shi, G., Flexural vibration and buckling analysis of orthotropic plates by the boundary element method. International Journal of Solids and Structures, 26(12), pp. 1351-1370, 1990.

[9] Cherukunnath, N. and Syngellakis, S., A boundary element approach to buckling of laminated plates subjected to arbitrary in-plane loading. Papers-AIAA, pp. 866-874, 2002.

[10] Syngellakis, S. and Cherukunnath, N., Boundary element modelling of nonlinear buckling for symmetrically laminated plates. Boundary Elements XXXI, ed. C.A. Brebbia, WIT Press: Southampton, pp. 211-222, 2009.

[11] Hwu, C., Green's function for the composite laminates with bending extension coupling. Composite Structures, 63, pp. 283-292, 2004.

[12] Hwu, C., Boundary integral equations for general laminated plates with coupled stretching-bending deformation. Proceedings of the Royal Society of London, Series A (Mathematical, Physical and Engineering Sciences), 466(2116), pp. 2010.

[13] Budiansky, B., Theory of buckling and post-buckling behavior of elastic structures. Advances in Applied Mechanics, ed. C.S. Yih, Academic Press: New York, pp. 1-65, 1974.

[14] Perez, M.M. and Wrobel, L.C., An integral equation formulation for anisotropic elastostatics. Journal of Applied Mechanics-Transactions of the ASME, 63(4), pp. 891-902, 1996.

[15] Bui, H.D., Some remarks about the formulation of three-dimensional thermoelastoplastic problems by integral equations. International Journal of Solids and Structures, 14(11), pp. 935-939, 1978.

[16] Syngellakis, S. and Cherukunnath, N., Stability analysis of laminate plates by the boundary element method. Boundary Elements XXIV, ed. C.A. Brebbia, A. Tadeu and V. Popov, WIT Press: Southampton, pp. 197-206, 2002.

[17] Syngellakis, S. and Walker, A.C., Elastic local buckling of longitudinally stiffened cylinders. Stability Problems in Engineering Structures and Components, ed. T.H. Richards and P. Stanley, Applied Science Publishers: Barking, Essex, pp. 159-178, 1978.

[18] Elzein, A. and Syngellakis, S., High-order elements for the BEM stability analysis of imperfect plates. Advances in Boundary Elements, Vol. 3: Stress Analysis, ed. C.A. Brebbia and J.J. Connor, Springer-Verlag: Berlin, pp. 269-289, 1989. 